\title{
Density enhancements associated with equatorial spread $F$
}

\author{
J. Krall ${ }^{1}$, J. D. Huba ${ }^{1}$, G. Joyce ${ }^{2}$, and T. Yokoyama ${ }^{3}$ \\ ${ }^{1}$ Plasma Physics Division, Naval Research Laboratory, Code 6790, 4555 Overlook Ave., SW, Washington, D.C., \\ 20375-5000, USA \\ ${ }^{2}$ Icarus Research, Inc., P.O. Box 30780, Bethesda, MD 20824-0780, USA \\ ${ }^{3}$ Department of Earth and Atmospheric Sciences, Cornell University, Ithaca, NY, 14850, USA
}

Received: 4 September 2009 - Revised: 17 November 2009 - Accepted: 23 November 2009 - Published: 1 February 2010

\begin{abstract}
Forces governing the three-dimensional structure of equatorial spread- $F$ (ESF) plumes are examined using the NRL SAMI3/ESF three-dimensional simulation code. As is the case with the equatorial ionization anomaly (IA), density crests within the plume occur where gravitational and diffusive forces are in balance. Large $\boldsymbol{E} \times \boldsymbol{B}$ drifts within the ESF plume place these crests on field lines with apex heights higher than those of the background IA crests. Large poleward field-aligned ion velocities within the plume result in large ion-neutral diffusive forces that support these ionization crests at altitudes higher than background IA crest altitudes. We show examples in which density enhancements associated with ESF, also called "plasma blobs," can occur within an ESF plume on density-crest field lines, at or above the density crests. Simulated ESF density enhancements reproduce all key features of those that have been observed in situ.
\end{abstract}

Keywords. Ionosphere (Equatorial ionosphere; Ionosphereatmosphere interactions; Ionospheric irregularities)

\section{Introduction}

Equatorial spread $F$ (ESF), named for the "diffuse echoes from the F-region of the ionosphere received continuously at night in equatorial regions over a wide range of wavefrequency" (Booker and Wells, 1938), has been a subject of active research for the past 35 years (Haerendel, 1974; Ossakow, 1981; Kelley, 1989; Hysell, 2000). Most recently, sophisticated three-dimensional computer simulation models of ESF plumes, or "bubbles," have been developed (Huba et al., 2008; Retterer, 2009). Because the strong electron

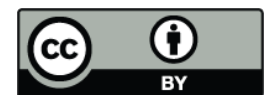

Correspondence to: J. Krall (jonathan.krall@nrl.navy.mil) density gradients associated with ESF affect the propagation of electromagnetic signals, sometimes degrading global communication and navigation systems (de La Beaujardiere et al., 2004; Steenburgh et al., 2008), the studies have continued. One of the many ESF-related phenomena that have yet to be explained is the occurrence of ESF density enhancements, also called "plasma blobs" (Oya et al., 1986; Watanabe and Oya, 1986; Le et al., 2003; Yokoyama et al., 2007; Park et al., 2008b; Martinis et al., 2009). These occur in regions of ESF activity, have been measured in situ by the Hinotori (altitude $\sim 650 \mathrm{~km})$, ROCSAT $(\sim 600 \mathrm{~km})$, DMSP $(\sim 840 \mathrm{~km})$, STSAT $(\sim 680 \mathrm{~km})$, and CHAMP $(\sim 350 \mathrm{~km})$ satellites, and may be related to the phenomena of ESF airglow enhancements (Krall et al., 2009b; Martinis et al., 2009). One aim of this paper is to provide an explanation for ESF density enhancements.

In recent past work with the SAMI3/ESF code (Huba et al., 2008) we have demonstrated and discovered several basic properties of the Rayleigh-Taylor-like ESF instability, such as the possible suppression of its growth by meridional winds (Krall et al., 2009a; Maruyama et al., 2009). We have found that ESF ion dynamics lead to specific compositional (Huba et al., 2009b) and thermal (Huba et al., 2009a) signatures. ESF-plume temperatures (Oyama et al., 1988; Park et al., 2008a) have been explained in some detail by Huba et al. (2009a), but many aspects of simulated ESF plume density structures have not.

For example, Huba et al. (2009b) showed that a "super fountain effect" within the ESF "bubble," or plume, leads to the development of ionization crests within the plume, similar to the ionization anomaly (IA) crests that usually occur in the post-sunset equatorial ionosphere. However, the forces governing these ionization crests have not yet been examined. In a related example, we found that a meridional wind can act in such a way that a density enhancement (relative to the nearby background) occurs within an ESF plume (Krall et al., 2009b) and that this enhancement has ion-velocity

Published by Copernicus Publications on behalf of the European Geosciences Union. 
profiles similar to those observed (Le et al., 2003; Yokoyama et al., 2007). In fact this same SAMI3/ESF study reproduced an observed ESF airglow phenomenon, ESF airglow enhancement, which is related to the above-mentioned ESF density enhancement (Krall et al., 2009b; Martinis et al., 2009). In more recent simulations, which we report below, we find that ESF density enhancements can occur in the absence of winds if the ESF plumes are growing relatively slowly.

In the present study, we use the SAMI3/ESF threedimensional simulation model to examine the 3-D structure of ESF plumes and the relationship between this structure and that of the background F-layer, particularly the IA crests. We further show examples of SAMI3/ESF simulations in which ESF density enhancements occur within the ESF plume, at or above these density crests. ESF-plume density structure is of interest because observations, both remote and in situ, show a wide variety of phenomena, including both density depletions, which are very common, and enhancements, which are less so (Watanabe and Oya, 1986; Le et al., 2003; Park et al., 2008b). Because we find that ESF plumes often include regions with ion densities equal to or greater than surrounding ionospheric densities, we will refer to them as "plumes" rather than "bubbles" in this study. As always, it is hoped that these simulations will aid in the interpretation and analysis of ESF measurements. To our knowledge, this is the first comprehensive modeling study of the forces governing the density structure within an ESF plume and the first simulation study of ESF density enhancements.

\section{SAMI3 simulations}

The NRL 3-D ionosphere code SAMI3/ESF (Huba et al., 2008 ) is used in this analysis. SAMI3/ESF is a version of the SAMI3 global ionosphere code (Huba et al., 2000, 2005) that has been modified for the study of ESF. SAMI3 models the plasma and chemical evolution of seven ion species $\left(\mathrm{H}^{+}\right.$, $\mathrm{He}^{+}, \mathrm{N}^{+}, \mathrm{O}^{+}, \mathrm{N}_{2}^{+}, \mathrm{NO}^{+}$and $\mathrm{O}_{2}^{+}$). The complete ion temperature equation is solved for three ion species $\left(\mathrm{H}^{+}, \mathrm{He}^{+}\right.$ and $\mathrm{O}^{+}$) as well as the electron temperature equation. The plasma equations solved are as follows:

$$
\begin{aligned}
& \frac{\partial n_{i}}{\partial t}+\nabla \cdot\left(n_{i} \boldsymbol{V}_{i}\right)=\mathcal{P}_{i}-\mathcal{L}_{i} n_{i} \\
& \frac{\partial \boldsymbol{V}_{i}}{\partial t}+\boldsymbol{V}_{i} \cdot \nabla \boldsymbol{V}_{i}=-\frac{1}{\rho_{i}} \nabla P_{i}+\frac{e}{m_{i}} \boldsymbol{E}+\frac{e}{m_{i} c} \boldsymbol{V}_{i} \times \boldsymbol{B}+\boldsymbol{g} \\
& -v_{i n}\left(\boldsymbol{V}_{i}-\boldsymbol{V}_{n}\right)-\sum_{j} v_{i j}\left(\boldsymbol{V}_{i}-\boldsymbol{V}_{j}\right) \\
& 0=-\frac{1}{n_{e} m_{e}} \nabla P_{e}+\frac{e}{m_{e}} \boldsymbol{E}+\frac{e}{m_{e} c} \boldsymbol{V}_{e} \times \boldsymbol{B} \\
& \frac{\partial T_{i}}{\partial t}+\boldsymbol{V}_{i} \cdot \nabla T_{i}+\frac{2}{3} T_{i} \nabla \cdot \boldsymbol{V}_{i}+\frac{2}{3} \frac{1}{n_{i} k} \nabla \cdot \boldsymbol{Q}_{i}=Q_{i n}+Q_{i i}+Q_{i e}
\end{aligned}
$$

$\frac{\partial T_{e}}{\partial t}-\frac{2}{3} \frac{1}{n_{e} k} b_{s}^{2} \frac{\partial}{\partial s} \kappa_{e} \frac{\partial T_{e}}{\partial s}=Q_{e n}+Q_{e i}+Q_{p h e}$

The various coefficients and parameters are specified in Huba et al. (2000). Ion inertia is included in the ion momentum equation for motion along the geomagnetic field and $\boldsymbol{E} \times \boldsymbol{B}$ drifts are computed to obtain motion transverse to the field. Neutral composition and temperature are specified using the empirical NRLMSISE00 model (Picone et al., 2002). The version of SAMI3 used here and in our other recent ESF studies (Huba et al., 2008, 2009a,b; Krall et al., 2009a,b) is modified relative to that used in past studies (Huba et al., 2005) in that the perpendicular electric field $\boldsymbol{E}_{\perp}=-\nabla \Phi$ is computed self-consistently in SAMI3 to determine the $\boldsymbol{E} \times \boldsymbol{B}$ drifts.

Of particular interest for this study is the field-aligned component of the ion momentum equation, which can be expressed as

$$
\begin{gathered}
\frac{\partial V_{i s}}{\partial t}+\left(\boldsymbol{V}_{i} \cdot \nabla\right) V_{i s}=-\frac{1}{n_{i} m_{i}} b_{s} \frac{\partial P_{i}}{\partial s}-\frac{1}{n_{i} m_{i}} b_{s} \frac{\partial P_{e}}{\partial s} \\
+g_{s}-v_{i n}\left(V_{i s}-V_{n s}\right)-\sum_{j} v_{i j}\left(V_{i s}-V_{j s}\right),
\end{gathered}
$$

where $s$ is the coordinate along the field line and where the electron momentum equation, Eq. (3), has been used to express the polarization electric field in terms of the electron pressure. SAMI3 uses a non-orthogonal, irregular grid that conforms to a model geomagnetic field. For this study the magnetic field is modeled as a dipole field aligned with the earth's spin axis.

The SAMI3/ESF potential equation is derived from current conservation $(\nabla \cdot \boldsymbol{J}=0)$ in dipole coordinates $(s, p, \phi)$ and is described in Huba et al. (2008). In this study the potential equation is identical to that of Krall et al. (2009b), except that the Hall-conductance terms are neglected relative to Pedersen-conductance terms (e.g., $\Sigma_{H}=\sigma_{H}=0$ ).

The 3-D simulation model is initialized using results from the two-dimensional SAMI2 code. SAMI2 is run for $48 \mathrm{~h}$ using the following geophysical conditions: $\mathrm{F} 10.7=75$, $\mathrm{F} 10.7 \mathrm{~A}=75, \mathrm{Ap}=4$, and day-of-year 80 (e.g., 21 March 2002). The simulation is centered at geographic longitude $0^{\circ}$ so universal time and local time are the same. The plasma is modeled from hemisphere to hemisphere up to $\pm 31^{\circ} \mathrm{mag}$ netic latitude; the peak altitude at the magnetic equator is $\sim 2400 \mathrm{~km}$. The $\boldsymbol{E} \times \boldsymbol{B}$ drift in SAMI2 is prescribed by the Fejer/Scherliess model (Scherliess and Fejer, 1999). The plasma parameters (density, temperature, and velocity) at time 19:20 UT (of the second day) are used to initialize the 3-D model at each magnetic longitudinal plane.

The 3-D model uses a grid with magnetic apex heights from $90 \mathrm{~km}$ to $2400 \mathrm{~km}$, and a longitudinal width of $4^{\circ}$ (e.g., $\simeq 460 \mathrm{~km})$. The grid is $\left(n_{z}, n_{f}, n_{l}\right)=(101,202,96)$ where $n_{z}$ is the number grid points along the magnetic field, $n_{f}$ is the number of "field lines," and $n_{l}$ is the number of longitude grid points. This grid has a resolution of $\sim 6 \mathrm{~km} \times 5 \mathrm{~km}$ in altitude 


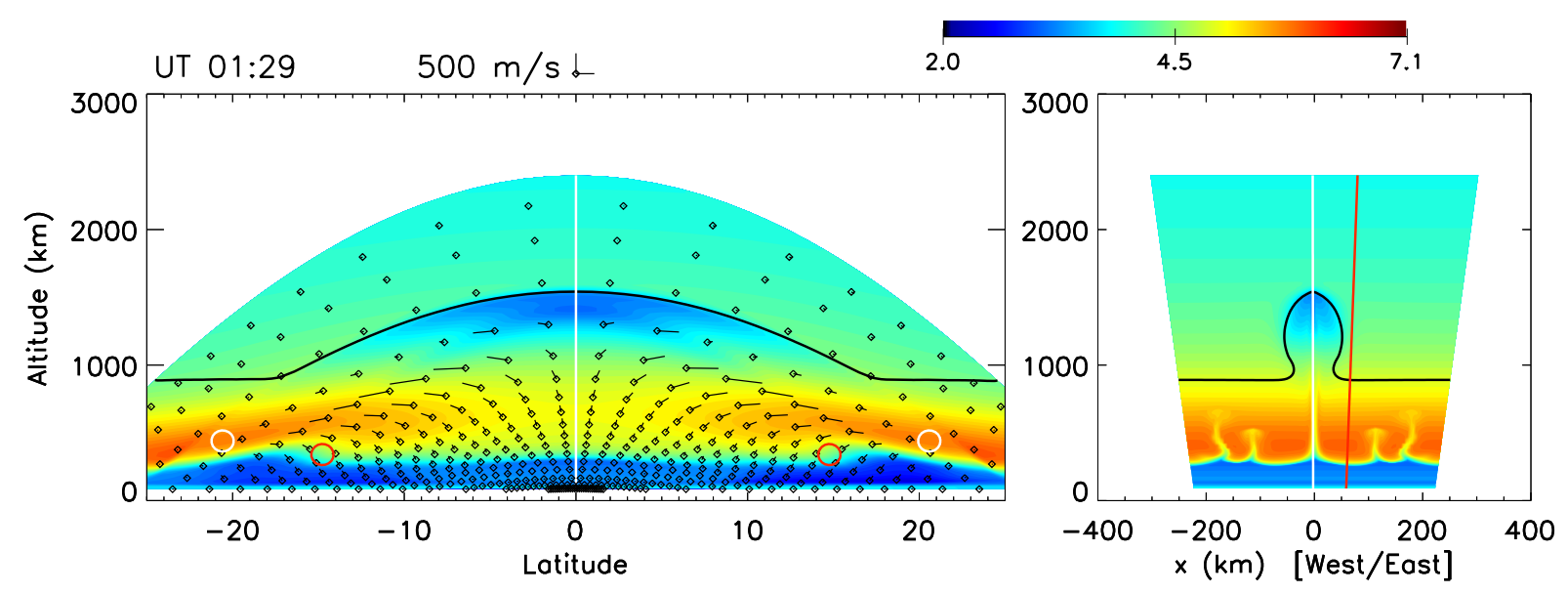

Fig. 1. Contours of $\log _{10} n_{e}$ at a fixed longitude (left) and at the magnetic equator (right). The location of the left-hand contour in the right-hand panel is indicated by a white line and vice versa. Ionization crests are indicated within the ESF plume (white circles) and in the background (red circles). A dark curve near the center of each plot indicates the $\mathrm{H}^{+} / \mathrm{O}^{+}$transition height. In the left hand panel, $\mathrm{O}^{+}$ion velocity vectors indicate the direction of flow away from the dot.

and longitude in the magnetic equatorial plane. The grid is periodic in longitude. In essence we are simulating a narrow "wedge" of the ionosphere in the post-sunset period. Unless noted, the ESF instability in each simulation was initiated by imposing a Gaussian-like perturbation in the ion density at $t=0$ : a peak ion density perturbation of $15 \%$ centered at $0^{\circ}$ longitude with a half-width of $0.25^{\circ}$, and at an altitude $z=300 \mathrm{~km}$ with a half-width of $50 \mathrm{~km}$. This seed perturbation extends along the entire flux tube. For this study, the vertical and zonal neutral winds are set to zero and the meridional neutral wind is set either to zero or to a constant value of $10 \mathrm{~m} / \mathrm{s}$.

\section{ESF density enhancements}

While ESF density depletions are common, both in the simulations and in a wealth of observations, density enhancements associated with ESF are seen less often (Watanabe and Oya, 1986; Le et al., 2003; Park et al., 2008b). We first describe the forces that support ionization crests within an ESF plume. We will then consider two situations in which the relatively high densities at or above these ionization crests can become ESF density enhancements.

\subsection{Ionization crests with an ESF plume}

Figure 1 shows an example of a simple ESF plume viewed in the plane of the magnetic equator (right panel) and in a constant longitude plane (left panel). In this case there are no winds $\left(V_{n s}=0\right)$. Contour plots show values of $\log _{10}\left(n_{e}\right)$, with the location of the left (right) contour plot being indicated by a white line in the right-hand (left-hand) panel. The edge of the ESF plume, which has an apex height of $1610 \mathrm{~km}$, can be seen in both panels as a discontinuity in the density. The left-hand panel shows the flow of $\mathrm{O}^{+}$ions, where velocity vectors indicate the direction of flow away from the dot in each case. A dark curve near the center of each plot indicates the $\mathrm{H}^{+} / \mathrm{O}^{+}$transition height, where the densities of these two ionospheric constituents are equal. That the bulk of the ESF plume lies below this transition height at all times is indicative of the transport of low-altitude-composition plasma to high altitude, as found by Huba et al. (2009b).

As was seen in past studies (Huba et al., 2008, 2009a,b; Krall et al., 2009a,b), the ESF plume is strongly depleted near its apex, $\boldsymbol{E} \times \boldsymbol{B}$ drifts move ions upward across field lines, gravity moves ions downwards along field lines (Hanson and Bamgboye, 1984), and a pair of ionization crests form within the ESF plume, here at altitude $440 \mathrm{~km}$ and latitude $\pm 21^{\circ}$. These are marked in the left-hand panel by white circles.

For comparison to the ionization crests within the ESF plume, we considered the properties of the background IA at a position indicated by the red vertical line in the righthand panel of Fig. 1. This is outside of the region affected by the localized electric potential of the nearby ESF plume. The position of the two background IA crests are indicated by red circles in the left-hand panel. We see that the ESF crests occur at an altitude above that of the background IA crests. Further, the ESF crests lie on a field line with an apex altitude above that of the background IA. In Fig. 1 (left panel) field lines are indicated by the black dots at the base of each vector; these dots are arrayed along selected field lines.

Past studies of the "fountain effect" that leads to the IA (Hanson and Moffett, 1966; Anderson, 1973) show that the crest positions are dependent on the magnitude of the upward $\boldsymbol{E} \times \boldsymbol{B}$ drift: a larger drift results in the IA crests occurring 


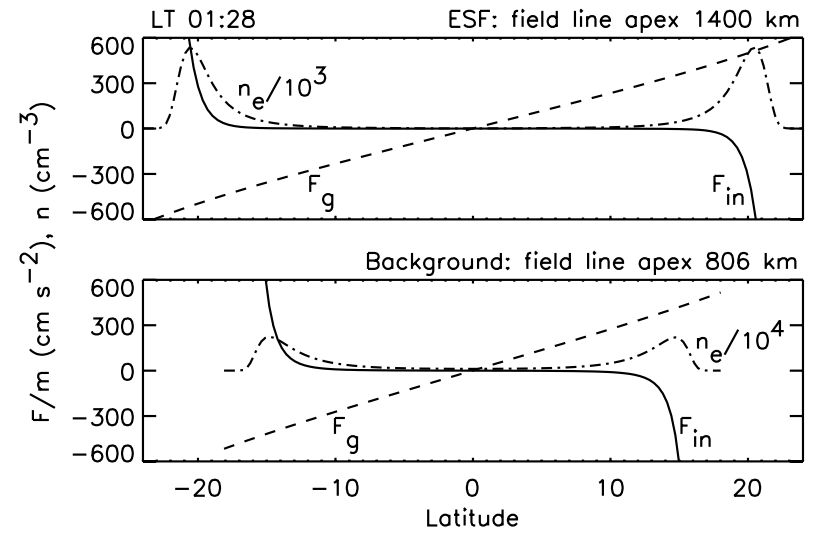

Fig. 2. Field-aligned gravity $\left(F_{g}\right.$, dashed line) and diffusion $\left(F_{i n}\right.$, solid) forces acting on $\mathrm{O}^{+}$ions are indicated within the ESF plume (top panel) and in the background ionosphere (bottom panel) for field lines corresponding to the ionization crests in each case. Electron density (dot-dash) is also plotted.

on geomagnetic field lines of higher altitude. These studies have also shown that the position of the IA crests along the geomagnetic field is governed by a balance between the downward gravitational force and the upward diffusion force that is exerted by neutrals on the downward-moving ions (see Eq. 6). This is illustrated in Fig. 2, where the electron density (dot-dash line) is plotted along with two of the forces acting on $\mathrm{O}^{+}$ions: the acceleration due to gravity $F_{g} / m_{i}=g_{s}$ (dashed) and the acceleration due to ion-neutral diffusion, $F_{\text {in }} / m_{i}=-v_{\text {in }} V_{i s}$ (solid). These forces are plotted versus latitude for the field line and longitude corresponding to the ESF crests (top panel) and for the background IA crests (bottom panel). In both cases, we see that the peak in the density occurs where the gravitational and diffusive forces are approximately equal in magnitude. To our knowledge, this is the first demonstration that the IA crests do indeed peak where the gravitational and diffusive forces are equal and opposite.

A more detailed look at the forces acting on $\mathrm{O}^{+}$ions within the ESF plume is shown in Fig. 3, where each of the five terms on the right-hand side of Eq. (6) is plotted in the upper panel. As in Fig. 2 (top), the plot is along the field line corresponding to the ionization crests within the ESF plume shown in Fig. 1. The lower panel of Fig. 3 shows the net force and the electron density. That the ionization crests within the ESF plume are held in place primarily by the downward gravity $\left(F_{g}\right)$ and upward diffusion $\left(F_{i n}\right)$ forces is confirmed in Fig. 3 (top), which shows that the ion and electron pressures are approximately zero where the electron density peaks and that the ion-ion diffusion force $F_{i j}$ is everywhere small. Figure 3 (bottom) shows that the ionization crests are in a nearequilibrium state, with the net force being close to zero in the crest regions.

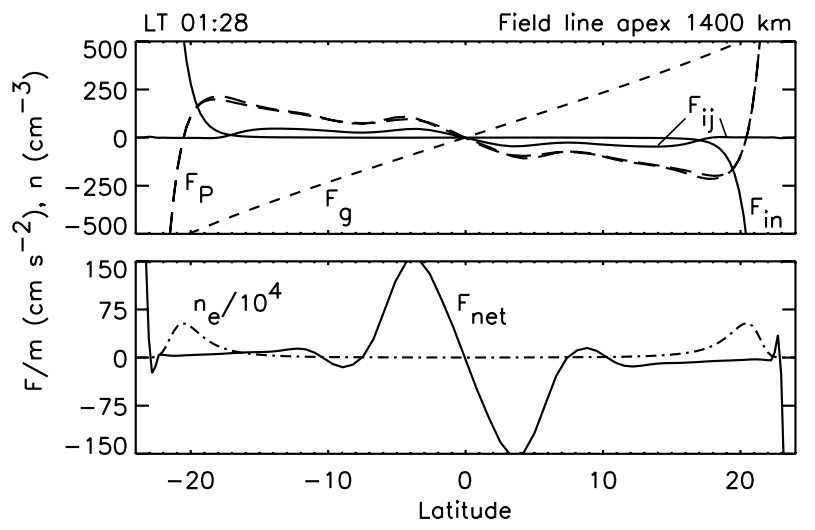

Fig. 3. Top panel: field-aligned forces, gravity (dashed line), ion and electron pressures (long-dashed lines), and diffusion $\left(F_{i n}, F_{i j}\right.$, solid lines), acting on $\mathrm{O}^{+}$ions are indicated for a field line corresponding to the ionization crests within the plume. Bottom panel: net field-aligned force (solid line) acting on $\mathrm{O}^{+}$ions is plotted. Electron density (dot-dash) is also shown.

These results further demonstrate the workings of the ESF "super fountain," which was first identified by Huba et al. (2009b). Through this and other three-dimensional ESF simulations, we find that the large $\boldsymbol{E} \times \boldsymbol{B}$ drifts within the ESF plume create ionization crests on field lines above those of the background IA crests. We also find that the large poleward field-aligned ion velocities within the plume result in large ion-neutral diffusive forces that support these ionization crests at altitudes above those of the background IA crests. The diffusive force is interesting in that it is only significant when ion (or neutral) velocities are large. However, we shall see in the next section that even a weakly growing ESF plume can generate velocities of $100-200 \mathrm{~m} / \mathrm{s}$ and that these are large enough to support ionization crests at altitudes higher than those of the background IA crests. Further results (not shown) show that $F_{i n}$ is dominated by collisions between $\mathrm{O}^{+}$and $\mathrm{O}$; when the contribution from this interaction is removed, the crests appear at a much lower altitude. The importance of $\mathrm{O}^{+} / \mathrm{O}$ collisions raises the possibility that the "Burnside factor" (Jee et al., 2005), which may increase the $\mathrm{O}^{+} / \mathrm{O}$ collisions rate by a factor as large as 1.8 , may have some effect. However, because $F_{\text {in }}$ varies so rapidly at the crest altitude (see Fig. 2), we find that this factor is of little importance to the position of the crests. Similarly, we considered the effect of of ion heating within the plume, which was found by Huba et al. (2009a). This was also found to be of little consequence. Indeed, the most significant factor in determining the height of the crests appears to be the downward, field-aligned ion velocity: larger velocities lead to higher crest altitudes.

While our analysis shows that the ion-ion diffusive force $F_{i j}$ is of little consequence to the positions of ionization crests, it is nevertheless interesting, with effects that may be 


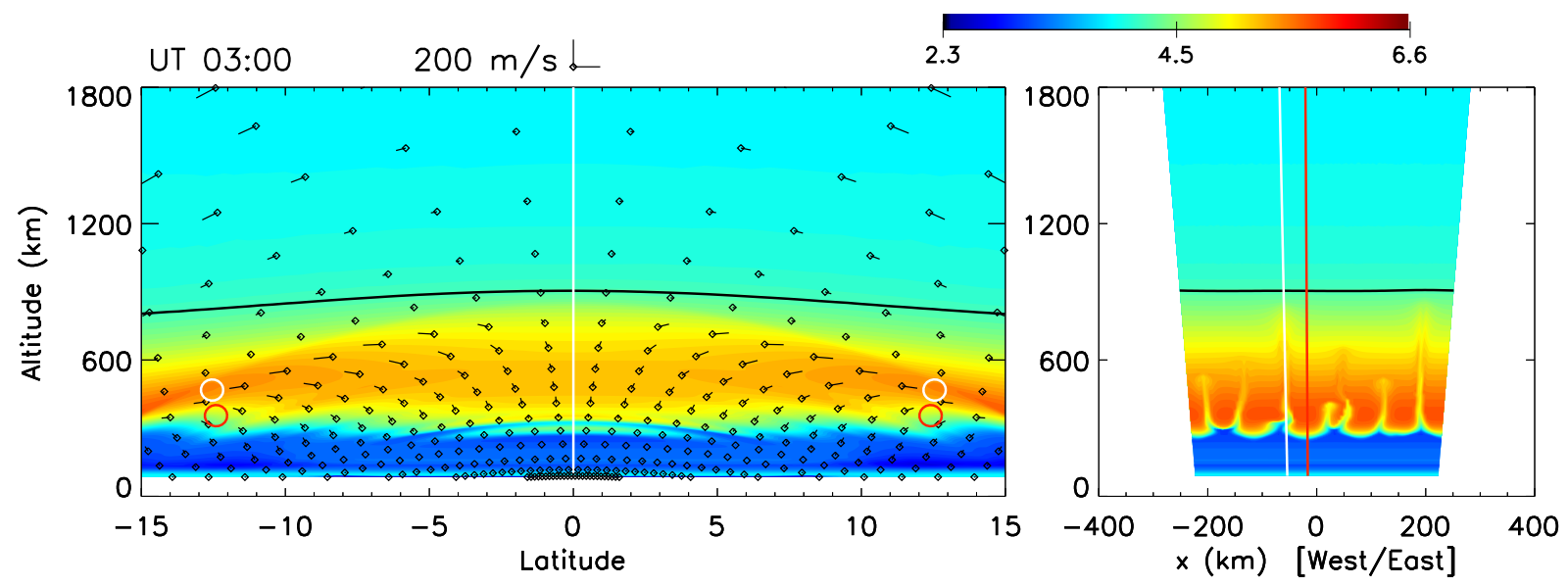

Fig. 4. Contours of $\log _{10} n_{e}$ at a fixed longitude (left) and at the magnetic equator (right). The location of the left-hand contour in the right-hand panel is indicated by a white line and vice versa. Ionization crests are indicated within the ESF plume (white circles) and in the background (red circles). A dark curve near the center of each plot indicates the $\mathrm{H}^{+} / \mathrm{O}^{+}$transition height. In the left hand panel, $\mathrm{O}^{+}$ion velocity vectors indicate the direction of flow away from the dot.

observable in situ. As implied by Huba et al. (2009b), $F_{i j}$ within the upper part of plume is dominated by the interplay between $\mathrm{O}^{+}$and $\mathrm{H}^{+}$ions, with the downward flow of the $\mathrm{O}^{+}$ "super fountain" initially dragging $\mathrm{H}^{+}$downward and out of hydrostatic equilibrium, followed by oscillations in the $\mathrm{H}^{+}$ velocity and, consequently, $F_{i j}$. Figure 3 (top) shows $F_{i j}$, the force exerted by $\mathrm{H}^{+}$on $\mathrm{O}^{+}$, during a phase in which $\mathrm{H}^{+}$ is moving upwards. Oscillations in the $\mathrm{H}^{+}$density also affect the ion and electron pressure terms near the apex of the plume.

\subsection{Density enhancements associated with weakly grow- ing ESF}

We now consider a case in which ESF develops with a low growth rate and without the occurrence of a strong super fountain (Huba et al., 2009b) within the ESF plume. Specifically, we consider a simulation where ESF plumes grow from random fluctuations in the ion density, temperature, and velocity instead of from a "seed" perturbation as in Fig. 1. The fluctuations were applied to the initial conditions only and were given characteristic amplitudes that varied versus height in such a way that fluctuation amplitudes everywhere correspond to the observed amplitudes of traveling ionospheric disturbances (TIDs) as reported in Hocke and Schlegel (1996, see Fig. 17 therein). For simplicity, the fluctuations were applied only to $\mathrm{O}^{+}$. Initial fluctuations are consistent with the TID observations only in terms of fluctuation amplitude versus height; we did not otherwise mimic TIDs. Outside of the height range of the reported observations, fluctuation amplitude versus height falls smoothly to zero such that there are no fluctuations below $50 \mathrm{~km}$ or above $900 \mathrm{~km}$. This simulation, shown in Fig. 4, is otherwise identical to that shown in Fig. 1.
Figure 4 (right) shows that several ESF plumes grow within the simulation. Figure 4 (left) shows density $\left(\log _{10} n_{e}\right)$ contours and velocity vectors within one of the ESF plumes. As in Fig. 1, the location of the left (right) contour plot is indicated by a white line in the right-hand (left-hand) panel. As in Fig. 1, ionization crests within the ESF plume are marked by white circles and the position of the background IA crests are marked by red circles. We see that, once again, the ESF ionization crests occur on a field line above that of the background IA crests and at an altitude above the background IA. The left-hand panel also shows the flow of $\mathrm{O}^{+}$ions, where velocity vectors indicate the direction of flow away from the dot in each case.

This simulation includes several interesting features. Because ESF is not a simple local instability (Rishbeth, 1971; Zalesak et al., 1982), ESF growth is dependent on the specifics of the three-dimensional initial perturbation. That ESF growth rates depend on field-line integrated quantities such as the Pedersen conductivity was verified by Krall et al. (2009a), who computed these growth rates for comparison to SAMI3/ESF simulation results. In fact, properties of the optimum naturally-occurring ESF seed have not yet been determined. For this TID-like initial perturbation, we find that ESF plumes grow more slowly than the "seeded" plume of Fig. 1 above, which reached a very high altitude as a result of the artificially-large initial density perturbation (15\%). At 03:00 local time the plume of Fig. 4 (left) has only reached an apex height of $850 \mathrm{~km}$. We emphasize that the initial seed perturbation is the only difference between these two cases.

Unlike typical ESF plumes, this plume contains a density enhancement near the edge of the plume at all heights above the locations of the ionization crests. Below the crests within the ESF plume the density is depleted relative to the background, as usual. This can be seen in Fig. 4 (left), where 


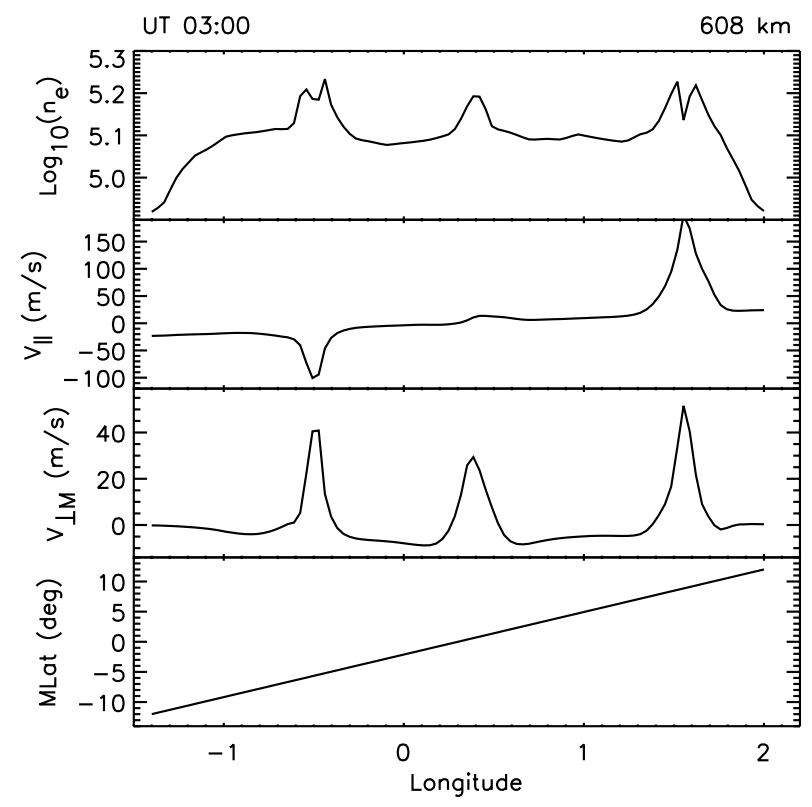

Fig. 5. Plots of $\log _{10} n_{e}$, field-aligned $\mathrm{O}^{+}$velocity $\left(V_{\|}\right.$, positive $=$ northward), vertical $\boldsymbol{E} \times \boldsymbol{B}$ velocity $\left(V_{\perp M}\right)$, and magnetic latitude versus longitude along a trajectory fixed altitude $608 \mathrm{~km}$.

the edge of the ESF plume is indicated by a discontinuity in $\log _{10}\left(n_{e}\right)$. Figure 4 (right) shows that the density is enhanced relative to the background only near the top of each plume (at altitudes of 600 to $850 \mathrm{~km}$ for the plume cross-section shown in the left-hand panel).

In fact, the simulation shows multiple plumes, all with density enhancements at these heights. The three tallest ESF plumes, all with apex heights above $600 \mathrm{~km}$, can be seen in Fig. 4 (right), and in Fig. 5. This figure shows plots of $\log _{10} n_{e}$, field-aligned $\mathrm{O}^{+}$velocity, vertical $\boldsymbol{E} \times \boldsymbol{B}$ velocity, and magnetic latitude versus longitude along a trajectory at fixed altitude $608 \mathrm{~km}$. The top panel shows density increases similar to those seen in satellite data (Oya et al., 1986; Le et al., 2003; Yokoyama et al., 2007; Park et al., 2008b; Martinis et al., 2009). We see that the $\boldsymbol{E} \times \boldsymbol{B}$ drifts are everywhere upward and that the field aligned flows are everywhere poleward. These are also characteristic of the satellite data (comparisons to the data will be discussed further below).

As in the case shown in Fig. 1, the density crests are held up by the ion-neutral diffusive force acting against the gravity-driven downward ion flow. The key to this result appears to lie in the relative weakness in the ESF super fountain in this case (note the change in the scale for the velocity vectors in Fig. 4 versus Fig. 1). The relatively weak seed, combined with effect of the low F10.7 index (F10.7=75 in all runs), leads to a plume in which a strong depletion at high altitude is never obtained. The field-aligned velocities are nevertheless strong enough that the upward diffusion force is able to support both the ESF ionization crests and the en- hanced densities that extend upwards along the ionizationcrest field lines to their apex. In our simulations to date, this situation has only occurred for low values $(<100)$ of the F10.7 index.

\subsection{Density enhancements associated with meridional winds}

We now consider the situation in which a meridional wind alters the plume and background densities in such a way that one of the ionization crests shown in Fig. 1 above becomes a density enhancement. Figure 6 shows a simulation with parameters identical to that of Fig. 1, but with a constant meridional wind of $10 \mathrm{~m} / \mathrm{s}$. Similar to the model results shown in Krall et al. (2009b, see Figs. 4 and 5 therein) this wind is strong enough to introduce a north-south asymmetry into the background ionospheric density while being too weak to suppress the growth of ESF (Krall et al., 2009a). In the case of Fig. 6, however, the EUV indices and the wind speed are much lower than in Krall et al. (2009b), where $\mathrm{F} 10.7=\mathrm{F} 10.7 \mathrm{a}=170$ and $V_{n}=20 \mathrm{~m} / \mathrm{s}$ were used.

Figure 6 shows the ESF plume in a constant-longitude plane (left panel). As expected from past theoretical (Maruyama, 1988; Zalesak and Huba, 1991; Sultan, 1996) and numerical efforts (Sultan, 1996; Krall et al., 2009a), the wind reduces the growth of the ESF plume relative to the case with no wind (Fig. 1). Here contour plots show values of $\log _{10}\left(n_{e}\right)$, with the location of the left (right) contour plot being indicated by a white line in the right-hand (left-hand) panel. The left-hand panel also shows the flow of $\mathrm{O}^{+}$ions, where velocity vectors indicate the direction of flow away from the dot in each case. As in Figs. 1 and 4 above, ionization crests within the ESF plume (white circles) occur at an altitude and on a field line above those of the background IA crests (red circles). Plots (not shown) of forces acting along a field line corresponding to the ionization crests within the plume verify that, once again, ionization crests are located where the gravitational and diffusion forces are in balance.

The edge of the ESF plume, which has an apex height of $1090 \mathrm{~km}$, can be seen in the left-hand panel as a discontinuity in the density. As in Fig. 4, the density inside the plume is not always lower then the adjacent density outside the plume. In the northern "leg" of the plume, $12^{\circ} \mathrm{N}, 700 \mathrm{~km}$, the density is noticeably higher than the nearby density outside of the plume. The density enhancement can also be seen in the right-hand panel, which here shows contours of $\log _{10}\left(n_{e}\right)$ versus longitude and altitude along a surface that slices through the northern half of simulation; this surface is indicated by the white curve in the left-hand panel. At longitude 0 and at heights of $580-720 \mathrm{~km}$, the right-hand panel shows electron densities higher than those of the background at the same altitude. By contrast, the density in the southern leg of the plume is lower than that of the background.

Both the enhanced density in the northern leg and the north-south asymmetry in the plume and background 


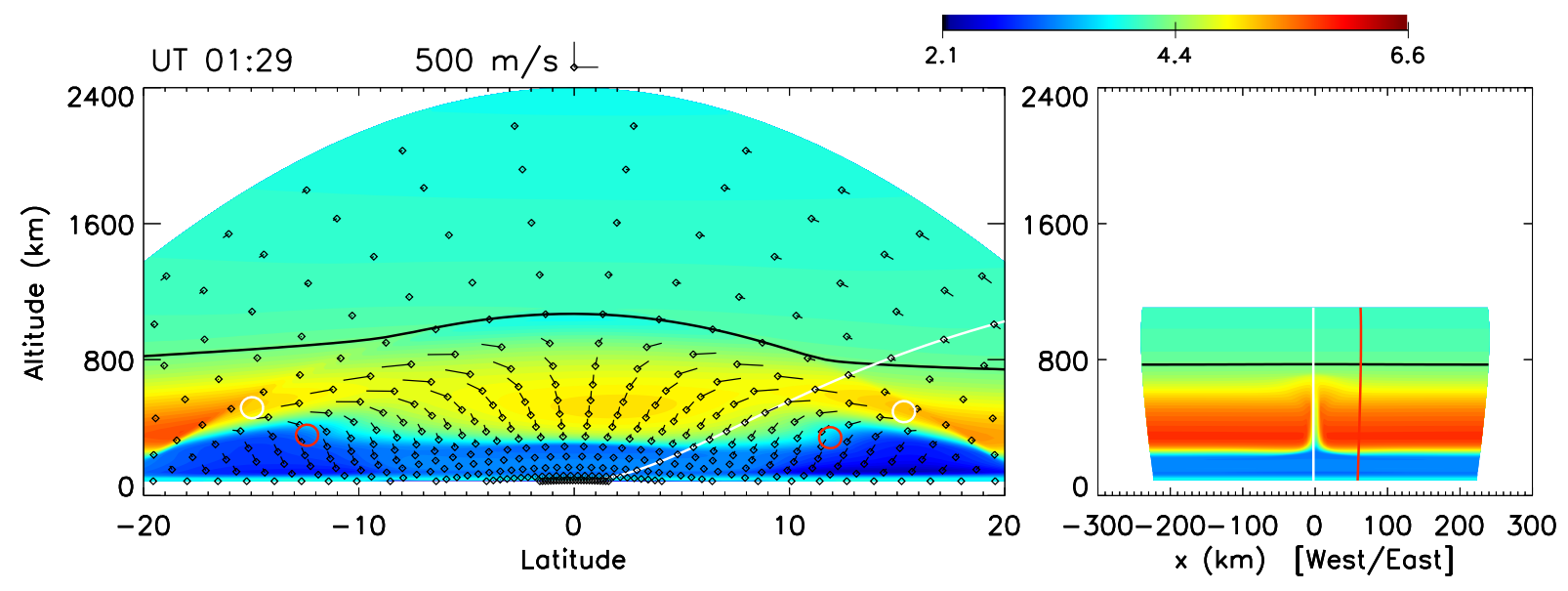

Fig. 6. Contours of $\log _{10} n_{e}$ at a fixed longitude (left) and in a surface that slices through the northern "leg" of the ESF plume (right). The location of the left-hand contour in the right-hand panel is indicated by a white line and vice versa. Ionization crests are indicated within the ESF plume (white circles) and in the background (red circles). A dark curve near the center of each plot indicates the $\mathrm{H}^{+} / \mathrm{O}^{+}$transition height. In the left hand panel, $\mathrm{O}^{+}$ion velocity vectors indicate the direction of flow away from the dot.

densities are evident in Fig. 7, which shows plots of $\log _{10} n_{e}$, field-aligned $\mathrm{O}^{+}$velocity, vertical $\boldsymbol{E} \times \boldsymbol{B}$ velocity, and magnetic latitude versus longitude along a trajectory at fixed altitude $590 \mathrm{~km}$. Note that while the format of this figure is similar to that of Fig. 5 above, the trajectory in this case is along a nearly-constant longitude such that it slices through both the northern and southern legs of the ESF plume shown in Fig. 6. In the top panel of Fig. 7 we see that the background electron density is about $10^{5} \mathrm{~cm}^{-3}$ at latitude $-15^{\circ}$, that the density drops at the southern edge of the plume (the interior of the plume is indicated by large field-aligned and upward velocities), that the density climbs to about $10^{5} \mathrm{~cm}^{-3}$ inside the northern leg of the plume (latitude $15^{\circ}$ ), and that the background density just outside of the northern leg of the plume drops to about $5 \times 10^{4} \mathrm{~cm}^{-3}$. Of the two peaks in the density curve, only the northern peak is a plasma blob; the southern peak is outside of the plume.

An interesting feature of Figs. 6 and 7 is that northsouth density asymmetry within the plume is opposite that of the north-south density asymmetry in the background ionosphere. As has been seen in past simulations and modeling, a northern wind tends to lift the southern IA crest, reducing its rate of recombination relative to that of the northern crest (e.g., see Fig. 5b of Sultan, 1996, or Fig. 2 of Krall et al., 2009a). As a result, the southern IA crest obtains a higher density. By contrast, within the ESF plume, the meridional wind appears to shift density from south to north, where it "piles up" at or above the northern ionization crest within the ESF plume. Our interpretation is that the ion density builds up in this region because it is at such a high altitude that significant recombination does not occur. The result, seen here and in Krall et al. (2009b, see Figs. 4 and 5 therein), is a density enhancement within the northern leg of the ESF plume.

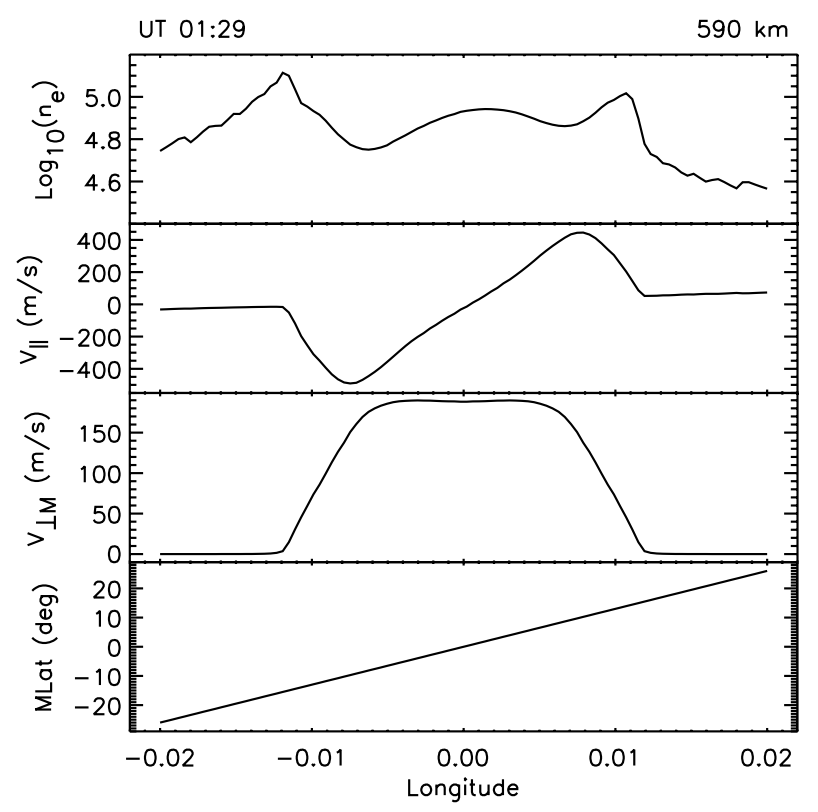

Fig. 7. Plots of $\log _{10} n_{e}$, field-aligned $\mathrm{O}^{+}$velocity $\left(V_{\|}\right.$, positive $=$ northward), vertical $\boldsymbol{E} \times \boldsymbol{B}$ velocity $\left(V_{\perp M}\right)$, and magnetic latitude versus longitude along a trajectory fixed altitude $590 \mathrm{~km}$.

As in Figs. 4 and 5 above, density enhancements are associated with upward $\boldsymbol{E} \times \boldsymbol{B}$ drifts and poleward field-aligned flows.

\subsection{Comparison to data}

Examples of in situ measurements of ESF density enhancements are shown in Figs. 8 and 9. These data come from 


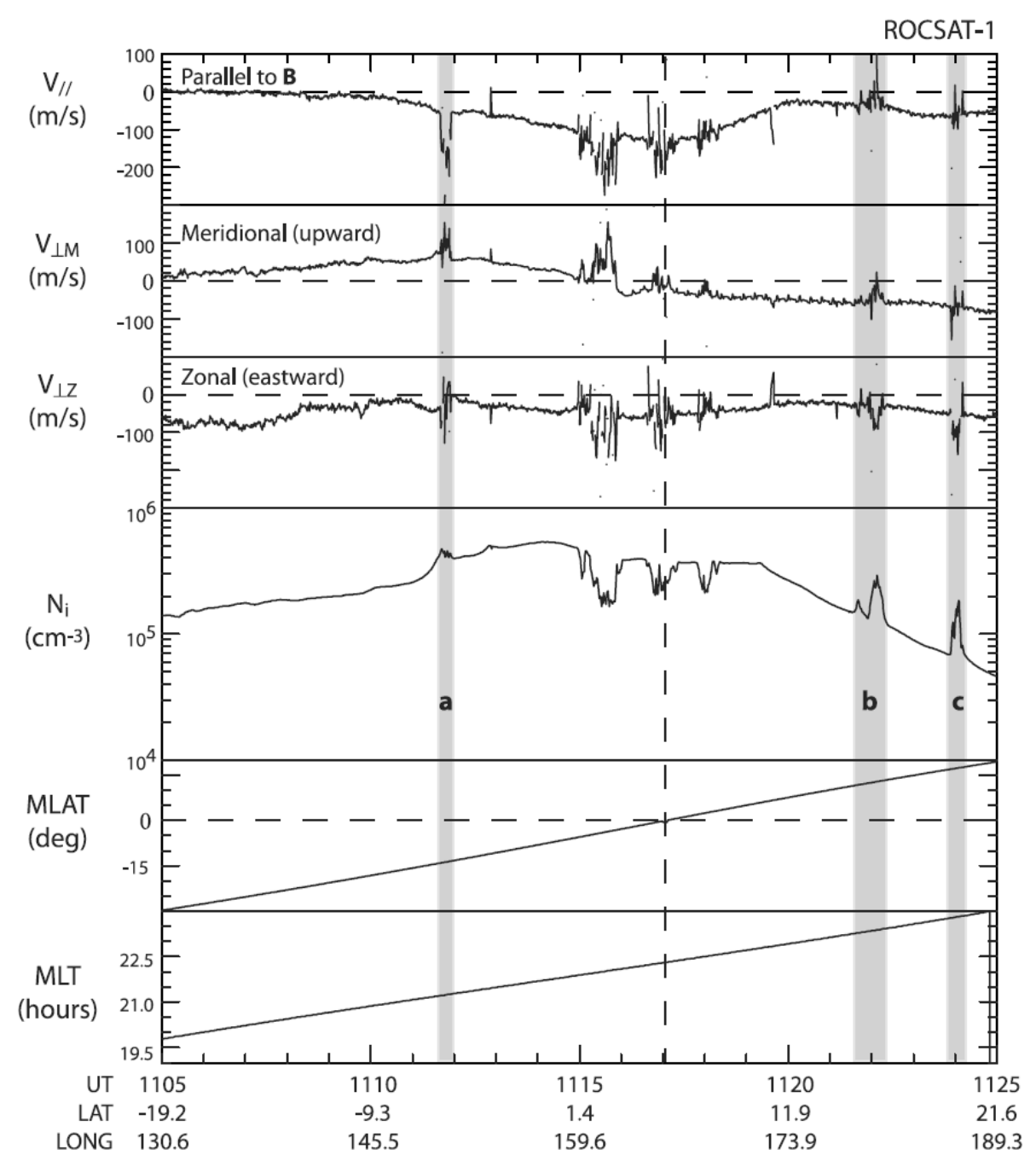

September 21, 1999 (DOY 264)

Fig. 8. Data from ROCSAT-1 at altitude $600 \mathrm{~km}$ on 21 September 1999: field-aligned ion velocity $V_{\|}$, vertical (meridional) perpendicular ion velocity $V_{\perp M}$, zonal perpendicular ion velocity $V_{\perp Z}$, electron density $N$, magnetic latitude, and local time plotted versus universal time and longitude. From Le et al. (2003).

the ROCSAT-1 satellite orbiting at an altitude of about $600 \mathrm{~km}$. Similar results have been obtained using the CHAMP $(350 \mathrm{~km})$, Hinotori $(650 \mathrm{~km})$, STSAT $(680 \mathrm{~km})$, and DMSP (F12, F14, and F15, 800-840 km) satellites, though neither Hinotori nor CHAMP include velocity data (Oya et al., 1986; Le et al., 2003; Park et al., 2008b).

In examining these data, we see features that are similar to those found in the simulation results of Figs. 5 and 7 above. In all cases, density enhancements represent increases of about $50-100 \%$ over background densities and ion velocities within the enhancements are upward and poleward. For example, similar to Fig. 8, Fig. 5 shows that the parallel ion velocity within the enhancements changes sign as the trajectory line crosses the magnetic equator. We also see, in Fig. 9, verification that density enhancements have a compositional signature (increased $\mathrm{O}^{+} / \mathrm{H}^{+}$ratio) that is characteristic of lower-altitude ionospheric plasma that has been lifted upwards as described by Huba et al. (2009b). Similar to Fig. 8, Fig. 7 shows that ESF-like depletions and enhancements occur simultaneously in the same region of the upper ionosphere. Simultaneous ROCSAT-1 and radar observations by Yokoyama et al. (2007) verify that density enhancements are not only associated with ESF, but that an enhancement in one hemisphere can co-exist with a depletion in the other on the same flux tube, as is the case in Fig. 7. Finally, similar to Fig. 9 and to the 7 January 2000 ROCSAT observation (Le et al., 2003, see Fig. 8 therein), Fig. 5 associates larger vertical $\left(V_{\perp M}\right)$ and field-aligned velocities with 


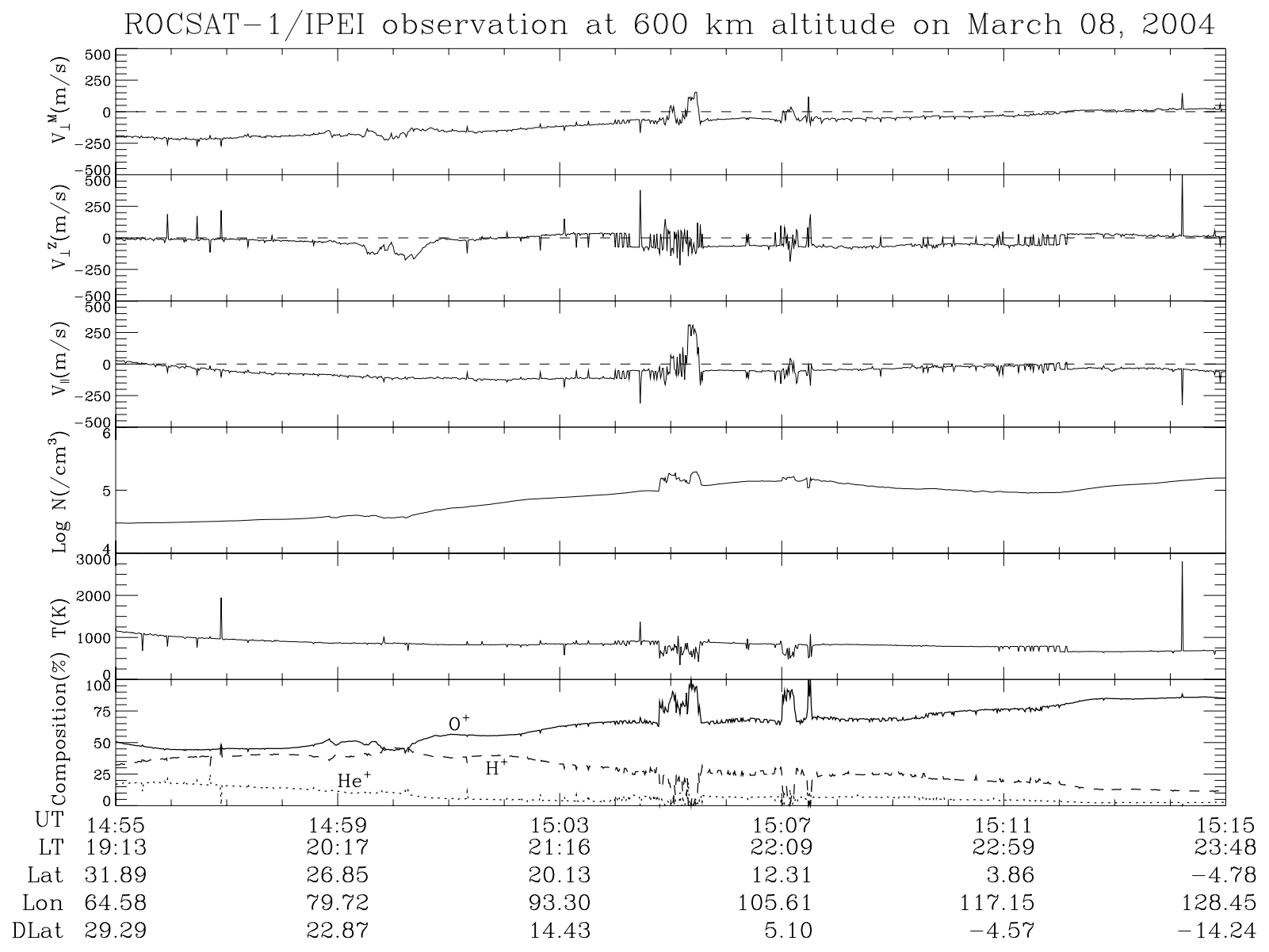

Fig. 9. Data from ROCSAT-1 at altitude $600 \mathrm{~km}$ on 8 March 2004: vertical (meridional) perpendicular ion velocity $V_{\perp}^{M}$, zonal perpendicular ion velocity $V_{\perp}^{Z}$, field-aligned ion velocity $V_{\|}$, electron density $\log N$, and $\mathrm{O}^{+}, \mathrm{H}^{+}$, and $\mathrm{He}^{+}$composition plotted versus universal time, longitude and dip latitude. From Yokoyama et al. (2007).

distances further from the magnetic equator (however, Fig. 8 does not). A more extensive study of density-enhancement data could determine whether or not this is a common pattern.

\section{Conclusions}

Using the NRL SAMI3/ESF three-dimensional simulation code, we have examined the forces governing the formation of the density crests that occur within an ESF plume as a result of the ESF "super fountain effect" (Huba et al., 2009b). As with the IA crests that usually occur in the post-sunset equatorial ionosphere, we find that ESF density crests are located where downward gravity and upward ion-neutral diffusive forces are in balance. We have shown that large $\boldsymbol{E} \times \boldsymbol{B}$ drifts within the ESF plume place these crests on field lines above those of the background IA crests in all cases. Similarly, large poleward field-aligned ion velocities within the plume result in large ion-neutral diffusive forces that support these ionization crests at altitudes higher than those of the background IA crests in all cases.

We have shown two circumstances in which the density on or above the ESF crests leads to a density enhancement, also called a "plasma blob," similar to those observed in situ (Oya et al., 1986; Watanabe and Oya, 1986; Le et al., 2003; Yokoyama et al., 2007; Park et al., 2008b; Martinis et al., 2009). In one case, slowly growing ESF produces upward $\boldsymbol{E} \times \boldsymbol{B}$ drifts that move density upward faster than gravity moves it downward, with the result that densities on ESFcrest field lines, at or above the density crests, have densities that are enhanced relative that of the background ionosphere. In this case the ESF super fountain is relatively weak, with velocities of order $200 \mathrm{~m} / \mathrm{s}$. In our simulations to date, this situation has only occurred for low values $(<100)$ of the F10.7 index.

The second circumstance in which an ESF density crest becomes a density enhancement occurs in the presence of a mild meridional wind. Because the ESF crests occur at a 
relatively high altitude, a meridional wind has an effect on the ESF crests that differs from its effect on the IA crests. Whereas a northward wind raises the southern IA crest, reducing recombination, and lowers the northern crest, enhancing recombination, a meridional wind acting on an ESF plume simply pushes plasma northward, causing a slight increase in the density of the northern crest (because the ESF crests occur at relatively high altitude, they are not strongly affected by recombination). As a result, in the presence of a mild northward meridional wind $(10 \mathrm{~m} / \mathrm{s}$ in this case), the northern ESF crest can become a density enhancement. Because we have simulated this phenomenon for a variety of circumstances (e.g. Fig. 5 of Krall et al., 2009b), we speculate that this may be a relatively common occurrence. Watanabe and Oya (1986) report that plasma blobs occur most often at a distance of $20-30^{\circ}$ away from the magnetic equator, which is consistent with our results with a non-zero meridional wind. In addition, both Watanabe and Oya (1986) and Park et al. (2008b) report a higher occurrence of blobs in the winter hemisphere, a circumstance which favors a transequatorial meridional wind.

Finally we have shown that the simulated density enhancements have properties similar to that of observed density enhancements, also called "plasma blobs" (Oya et al., 1986; Le et al., 2003; Yokoyama et al., 2007; Park et al., 2008b; Martinis et al., 2009). In all cases, density enhancements represent increases of 50-100\% over background densities as observed. Further, ion velocities within the enhancements are upward and poleward in all cases, as is typically observed (Le et al., 2003; Yokoyama et al., 2007; Martinis et al., 2009). We note that observed blobs show a compositional signature (increased $\mathrm{O}^{+} / \mathrm{H}^{+}$ratio, Yokoyama et al., 2007) that is characteristic of lower-altitude ionospheric plasma that has been lifted upwards, as in our simulations (Huba et al., 2009b). As is seen in the observations, our simulations show that ESF-like depletions and enhancements occur simultaneously in the same region of the upper ionosphere (Oya et al., 1986; Watanabe and Oya, 1986; Le et al., 2003; Park et al., 2008b; Martinis et al., 2009). This association of density enhancements and ESF activity has been further verified by Yokoyama et al. (2007), who report simultaneous ROCSAT1 and radar observations showing that an enhancement in one hemisphere can co-exist with a depletion in the other hemisphere on the same flux tube (see also Fig. 7 above), and Martinis et al. (2009), who show that density enhancements are co-located on the same field lines as ESF airglow enhancements (see also Krall et al., 2009b).

We conclude, consistent with Le et al. (2003) and Martinis et al. (2009), that these density enhancements are signatures of ESF. However, we find that the enhanced densities are supported by the enhanced ion-neutral diffusive forces that are associated with the high field-aligned ion velocities of the ESF super fountain rather than a wind-driven instability in the topside ionosphere, as suggested by Watanabe and Oya (1986), or pressure forces, as suggested by Le et al. (2003).
We further assert that, while ESF plume densities are generally much lower than surrounding ionospheric densities, typical ESF plumes nevertheless contain regions in which the plume density is close to or greater than that of the background ionosphere.

Acknowledgements. The authors would like to thank Sidney Ossakow of Berkeley Research Associates for helpful discussions. This work was supported by the Office of Naval Research and NASA.

Topical Editor K. Kauristie thanks two anonymous referees for their help in evaluating this paper.

\section{References}

Anderson, D. N.: A theoretical study of the ionospheric $F$ region equatorial anomaly-I. Theory, Planet. Space Sci., 21, 409-419, 1973.

Booker, H. G. and Wells, H. W.: Scattering of radio waves in the $F$-region of ionosphere, Terr. Mag. Atmos. Elec., 43, 249-256, 1938.

de La Beaujardiere, O., Jeong, L., and The C/NOFS Science Definition Team: C/NOFS: a mission to forecast scintillations, J. Atmos. Solar Terr. Phys., 66, 1573-1591, 2004.

Haerendel, G.: Theory of equatorial spread $F$, preprint, Max Planck Inst. Extraterr. Phys., Munich, Germany, 1974.

Hanson, W. B. and Bamgboye, D. K.: The measured motions inside equatorial plasma bubbles, J. Geophys. Res., 89, 8997-9008, 1984.

Hanson, W. B. and Moffett, R. J.: Ionization transport effects in the equatorial $F$ region, J. Geophys. Res., 71, 5559-5572, 1966.

Hocke, K. and Schlegel, K.: A review of atmospheric gravity waves and travelling ionospheric disturbances: 1982-1995, Ann. Geophys., 14, 917-940, 1996, http://www.ann-geophys.net/14/917/1996/.

Huba, J. D., Joyce, G., and Fedder, J. A.: SAMI2 (Sami2 is Another Model of the Ionosphere): A New Low-Latitude Ionosphere Model, J. Geophys. Res., 105, 23035-23053, 2000.

Huba, J. D., Joyce, G., Sazykin, S., Wolf, R., and Shapiro, R.: Simulation study of penetration electric fields in the low- to mid-latitude ionosphere, Geophys. Res. Lett., 32, L23101, doi: 10.1029/2005GL024162, 2005.

Huba, J. D., Joyce, G., and Krall, J.: Three-dimensional equatorial spread $F$ modeling, Geophys. Res. Lett., 35, L10102, doi:10. 1029/2008GL033509, 2008.

Huba, J. D., Joyce, G., Krall, J., and Fedder, J.: Ion and electron temperature evolution during equatorial spread-F, Geophys. Res. Lett., 36, L15102, doi:10.1029/2009GL038872, 2009a.

Huba, J. D., Krall, J., and Joyce, G.: Atomic and molecular ion dynamics during equatorial spread- $F$, Geophys. Res. Lett., 36, L10106, doi:10.1029/2009GL037675, 2009b.

Hysell, D. L.: An overview and synthesis of plasma irregularities in equatorial spread $F$, J. Atmos. Solar Terr. Phys., 62, 1037-1056, 2000.

Jee, G., Schunk, R. W., and Scherliess, L.: On the sensitivity of the total electron content (TEC) to upper atmospheric/ionospheric parameters, J. Atmos. Solar Terr. Phys., 67, 1040-1052, 2005.

Kelley, M. C.: The Earth's Ionosphere, Plasma Physics and Electrodynamics, Academic, San Diego, Calif., 1989. 
Krall, J., Huba, J. D., Joyce, G., and Zalesak, S. T.: Threedimensional simulation of equatorial spread-F with meridional wind effects, Ann. Geophys., 27, 1821-1830, 2009a, http://www.ann-geophys.net/27/1821/2009/.

Krall, J., Huba, J. D., and Martinis, C.: Three dinensional modeling of equatorial spread- $F$ airglow enhancements, Geophys. Res. Lett., 36, L10103, doi:10.1029/2009GL038441, 2009b.

Le, G., Huang, C.-S., Pfaff, R. F., Su, S.-Y., Yeh, H.-C., Heelis, R. A., Rich, F. J., and Hairston, M.: Plasma density enhancements associated with equatorial spread $F$ : ROCSAT1 and DMSP observations, J. Geophys. Res., 108, 1318, doi:10.1029/2002JA009592, 2003.

Martinis, C., Baumgardner, J., Mendillo, M., Su, S.-Y., and Aponte, N.: Brightening of $630.0 \mathrm{~nm}$ airglow depletions observed at midlatitudes, J. Geophys. Res., 114, A06318, doi: 10.1029/2008JA013931, 2009.

Maruyama, T.: A diagnostic model for equatorial spread- $F 1$. Model description and application to electric field and neutral wind effects, J. Geophys. Res., 93, 14611-14622, 1988.

Maruyama, T., Saito, S., Kawamura, M., Nozaki, K., Krall, J., and Huba, J. D.: Equinoctial asymmetry of a low-latitude ionosphere-thermosphere system and equatorial irregularities: evidence for meridional wind control, Ann. Geophys., 27, 20272034, 2009, http://www.ann-geophys.net/27/2027/2009/.

Ossakow, S. L.: Spread $F$ theories: A review, J. Atmos. Terr. Phys., 43, 437-452, 1981.

Oya, H., Takahashi, T., and Watanabe, S.: Observation of low latitude ionosphere by the impedance probe on board the Hinotori satellite, J. Geomag. Geoelectr., 38, 111-123, 1986.

Oyama, K.-I., Schlegel, K., and Watanabe, S.: Temperature structure of plasma bubbles in the low latitude ionosphere around $600 \mathrm{~km}$ altitude, Planet. Space Sci., 36, 553-567, 1988.

Park, J., Min, K. W., Kim, V. P., Kil, H., Su, S.-Y., Chao, C. K., and Lee, J.-J.: Equatorial plasma bubbles with enhanced ion and electron temperatures, J. Geophys. Res., 113, A09318, doi:10. 1029/2008JA013067, 2008a.
Park, J., Stolle, C., Lühr, H., Rother, M., Su, S.-Y., Min, K. W., and Lee, J.-J.: Magnetic signatures and conjugate features of low-latitude plasma blobs as observed by the CHAMP satellite, J. Geophys. Res., 113, A09313, doi:10.1029/2008JA013211, 2008b.

Picone, J. M., Hedin, A. E., Drob, D. P., and Aikin, A. C.: NRLMSISE-00 empirical model of the atmosphere: Statistical comparisons and scientific issues, J. Geophys. Res., 107, 1468, doi:10.1029/2002JA009430, 2002.

Retterer, J. M.: Forecasting low-latitude radio scintillation with 3D ionospheric plume models: 1. Plume model, J. Geophys. Res., doi:10.1029/2008JA013839, in press, 2009a.

Rishbeth, H.: Polarization fields produced by winds in the equatorial $F$ region, Planet. Space Sci., 19, 357-369, 1971.

Scherliess, L. and Fejer, B. G.: Radar and satellite global equatorial $F$ region vertical drift model, J. Geophys. Res., 104, 6829-6842, 1999.

Steenburgh, R. A., Smithtro, C. G., and Groves, K. M.: Ionospheric scintillation effects on single frequency GPS, Space Weather, 6, S04D02, doi:10.1029/2007SW000340, 2008.

Sultan, P. J.: Linear theory and modeling of the Rayleigh-Taylor instability leading to the occurrence of equatorial spread $F$, J. Geophys. Res., 101, 26875-26891, 1996.

Watanabe, S. and Oya, H.: Occurence characteristics of low latitude ionosphere irregularities observed by impedance probe on board the Hinotori satellite, J. Geomag. Geoelectr., 38, 125-149, 1986.

Yokoyama, T., Su, S.-Y., and Fukao, S.: Plasma blobs and irregularities concurrently observed by ROCSAT-1 and Equatorial Atmosphere Radar, J. Geophys. Res., 112, A05311, doi: doi:10.1029/2006JA012044, 2007.

Zalesak, S. T. and Huba, J. D.: Effect of meridional winds on the development of equatorial spread- $F$, Eos Trans. AGU, 72, Spring Meet. Suppl., 211, 1991.

Zalesak, S. T., Ossakow, S. L., and Chaturvedi, P. K.: Nonlinear equatorial spread $F$ : The effect of neutral winds and background Pedersen conductivity, J. Geophys. Res., 87, 151-166, 1982. 